\title{
Admission patterns in a psychiatric intensive care unit in Ireland: a longitudinal follow-up
}

\author{
S. Raaj $^{1,2,3}$ (i) , S. Navanathan ${ }^{4,5}$, B. Matti ${ }^{6}$, A. Bhagawan1 ${ }^{1}$ P. Twomey ${ }^{1}$, J. Lally ${ }^{4,7,8,9,+}$ (i) and R. Browne ${ }^{1,4, \dagger}$ \\ ${ }^{1}$ Psychiatry Intensive Care Unit, Phoenix Care Centre, Dublin, Ireland \\ ${ }^{2}$ Department of Liaison Psychiatry, Mater Misericordiae University Hospital, Dublin 7, Ireland \\ ${ }^{3}$ Department of Psychiatry, School of Medicine, University College Dublin, Dublin, Ireland \\ ${ }^{4}$ Department of Psychiatry, Royal College of Surgeons in Ireland, Dublin, Ireland \\ ${ }^{5}$ Department of Psychiatry Old Age, Ashlin Centre, Beaumont Hospital, Dublin, Ireland \\ ${ }^{6}$ Department of Psychiatry, St. Patrick University Hospital, Dublin 8, Ireland \\ ${ }^{7}$ Department of Psychiatry, Mater Misericordiae University Hospital, Dublin 7, Ireland \\ 8 Department of Psychiatry, St. Vincent's Hospital Fairview, Dublin, Ireland \\ ${ }^{9}$ Department of Psychosis Studies, Institute of Psychiatry, Psychology and Neuroscience (IoPPN), King's College London, London, United Kingdom
}

Objective: This study aims to describe the course of admission and clinical characteristics of admissions to a psychiatric intensive care unit (PICU) in the Phoenix Care Centre (PCC), Dublin, Ireland.

Methods: This retrospective chart study was conducted at the PCC, Dublin, Ireland. The cohort included all admission episodes ( $n=91$ complete data) over a three-year study period between January 2014 and January 2017.

Results: The mean age of admitted cases was 37.1 (S.D. = 11.3; range 18-63). The mean length of stay (LOS) was 59.3 days (S.D. = 61.0; median 39.5 days). All patients were admitted under Mental Health Act legislation. Antipsychotic polypharmacy was used in $61 \%$ $(n=55)$ of the admissions. A diagnosis of acute psychotic disorder ( $\mathrm{B}=-1.027, p=0.003,95 \% \mathrm{CI}:-1.691,-0.363)$ was associated with reduced LOS in PICU.

Conclusion: Our study describes the cohort of patients admitted as being predominantly male, younger-aged, single, having a diagnosis of schizophrenia and being legally detained. The primary indication for referral is risk of assault, which highlights the need for the intensive and secure treatment model that a PICU can provide.

Received 10 March 2020; Revised 20 December 2020; Accepted 20 February 2021

Key words: High dose antipsychotic, length of stay, mental disorder, PICU, psychiatry intensive care unit.

\section{Introduction}

There is limited literature on psychiatric intensive care units (PICUs) in the Republic of Ireland. In the last three decades, psychiatric care in Ireland has transitioned from asylum care to a community mental health service model. This led to a reduction in the total number of patients in institutional care in Ireland. The Phoenix Care Centre (PCC) is a built for purpose facility that was built on the grounds of St. Brendan's Hospital as set out in the national policy and initiatives 'A Vision for Change' for mental health in 2006 (Health Service Executive, 2015; HSE, 2017). St. Brendan's Hospital opened in 1851 and is the first public psychiatric hospital developed in Ireland. PCC is a state-of-the-art purpose-built mental health facility which includes a PICU located in inner-city Dublin.

Address for correspondence: S. Raaj, Psychiatry Intensive Care Unit, Phoenix Care Centre, Dublin, Ireland.

(Email: Shaeraine@hotmail.com)

${ }^{\dagger}$ These author are Co-Senior authors.
A PICU is a tertiary mental health service designed to provide intensive care to patients who are in an acutely disturbed phase of a major mental disorder (Rachlin, 1973; Mounsey, 1979; Bowers et al. 2008; Bailey, 2014; Winkler et al. 2019). PICU units were first established in the 1970s in the UK (Mounsey, 1979) and United States (Rachlin, 1973) to manage patients who presented with behavioural disturbance, agitation and increased risk of harm to self or others. A previous systematic review described a typical PICU patient as likely to be young, male, single, unemployed, with a diagnosis of schizophrenia or mania, from the Caribbean or African background, legally detained with a forensic history (Bowers et al. 2008). The UK and Australian literature described PICU as small wards, with a higher proportion of nursing and other staff, built on an open plan locked ward designed to promote observation and often with facilities for seclusion (Bowers et al. 2008).

An early study published on PICUs by Rachlin (1973) from New York, described the unit as a 'closed ward' (Rachlin, 1973). The admission policy was rigid in that 
patients were only accepted from other psychiatric facilities after the referring ward had exhausted all other methods of management (Rachlin, 1973). A similar admission criterion was described by another PICU unit in New York in 1979. Colin and Jordan (1979) described the admission policy to be only accepting patients after 'every effort' had been supplemented to manage the patient in an open ward. Mounsey (1979) described the intensive care unit in the south of England as a 12-bedded unit established for patients who 'had presented serious management problem in their ward of origin' (Crowhurst \& Bowers, 2002; Goldney et al. 1985). These variations suggest a lack of standardising admission criteria to the PICU, and this might be explained by the difference in mental health legislation and policies in each country. The literature suggests that PICU units in the UK and abroad have a voluntary and involuntary admission criterion, with the majority of patients legally detained under the Mental Health Act (Crowhurst \& Bowers, 2002; Bowers et al. 2008; Winkler et al. 2019). However, in Ireland, the PICU at the PCC has restrictive admission criterion. The admission policy describes only accepting patients who are legally detained under the Mental Health Act 2001 and only after they 'cannot be safely assessed or treated in an open acute inpatient facility'. This shows dissimilarities exist between PICU's in different countries in terms of legal status, patient selection criteria, type of care and treatment outcome (Crowhurst \& Bowers, 2002; Karen Barlow et al. 2000; Bowers et al. 2008; Koppelmans \& Schoevers, 2009; Bailey, 2014; Winkler et al. 2019). It is unclear whether these differences in service operation and admission criteria of PICU's are built on community needs, or a result of historical or financial developments.

The majority of mental disorders are treated in the community, with only a small percentage requiring inpatient treatment (Kessler et al. 2005). These cases can present with an associated increased risk to self or others, which poses a challenge for therapeutic management and treatment in a less secure mental health ward (Bowers et al. 2008). The patient cohort admitted to a PICU requires higher intensity, more restrictive interventions to manage the increased risk (Karen Barlow et al. 2000; Bailey, 2014; Pararajasingam et al. 2017). The frequency of threatening and aggressive incidents differ with the phase of the psychiatric disorder (Vaaler \& Iversen, 2011). The highest rate of such incidents is described in the acute stage of inpatient hospital stay, which is the first few days of admission (Valeer \& Iversen, 2011).

\section{Aim}

This observational study aims to describe the course of the admission and clinical characteristics of admissions to the PICUs in the PCC, Dublin, Ireland. The authors hypothesised that the length of stay (LOS) would be shorter in male patients as compared to females.

\section{Methodology}

\section{Methods}

This retrospective cohort study was carried out at the PCC, Dublin, Ireland. Informed consent was not sought as this was a retrospective chart study involving anonymised clinical data which were collected as part of routine clinical care and no items of information were reported that would enable the identification of any subject.

\section{Clinical unit}

The PCC North Dublin is state of the art built for purpose psychiatric hospital that was opened in May 2013 (Health Service Executive, 2015). The inpatient service of a 54-bedded facility which cost approximately 21 million euros (Health Service Executive, 2015) and is divided into two separate units. The intensive care unit consists of a separate male and female ward constituting of 12 beds each, and there is a 30-bed rehabilitation unit divided into two wards. The two 12-bedded PICU on the ground floor is designed around a landscaped courtyard. Single ensuite bedrooms which open out to the central circular space which encircles a courtyard and a nursing station with good visibility. This facility includes an open dining room, activities room, television lounge, two quiet rooms, a seclusion room and open access courtyard on each ward. These designated areas provide a low-stimulus environment and open space for patients. An indoor gym was developed in 2017 for patients and is supervised by the Occupational Therapist at all times.

\section{Population}

The PICU at PCC, Dublin, Ireland, covers a population above 2 million inhabitants within the catchment areas of Dublin, Wicklow and the North-East Region (Health Service Executive, 2015). Ireland's Mental Health Services are publicly funded.

\section{Staffing}

The PCC relies on dynamic factors, for example, increased nurse to patient ratio and security. Static characteristics are locked doors, security cameras and walls to minimise risk (Bailey et al. 2014). Each ward is composed of a well-resourced specialist multidisciplinary team, consisting of a consultant psychiatrist, trainee psychiatrist, nursing, full-time mental health social worker, occupational therapist, clinical psychologist, pharmacist and a part-time dietician. A fully-staffed multidisciplinary team for a 12-bedded unit provides 
a higher level of support and increased face to face time with patients. Each ward is complemented with six nursing staff rostered during the day and four at night, and these are regularly supplemented by additional nurses when patients require further care.

\section{Admission criteria}

The admission policy states patients admitted/transferred to the PICU should be legally detained as an involuntary patient under the Mental Health Act 2001. The criteria for patient admission should be an immediate danger to themselves or others or pose a significant risk of absconding in the context of a major mental disorder. Patients are admitted to the PICU from another acute psychiatric facility (approved centres).

\section{Inclusion and exclusion criteria}

For the current study all admissions from an acute psychiatric unit to the PICU between January 2014 and Jan 2017 were eligible. Patients in the rehabilitation unit are excluded. The readmission of patients was also taken into account.

\section{Data collection}

All patients admitted between January 2014 and January 2017 to PCC formed the study group. Information for the study was extracted from clinical medical records and medication charts. Data collected from clinical medical records included a total of 14 variables: Gender, age, ethnicity, marital status, primary ICD 10 diagnosis, concurrent alcohol/substance use, behaviour precursor (behaviour preceding referral to the PICU), referral source, previous admission to PICU, forensic history, course of admission (number of restraint episodes, seclusion episodes, 1:1 nursing interventions), list of psychotropic medications (this includes antipsychotic dose in milligrams expressed as a percentage of the BNF maximum recommended daily dose), high antipsychotic dose (cumulative antipsychotic dose $>100 \%$ of maximum BNF recommended daily dose) and length of stay (LOS).

\section{Data analysis}

We described primary outcomes using frequencies, percentages, mean and standard deviations, median and interquartile ranges. Between groups comparisons were made using $x 2$ tests for categorical variables; $t$-tests, ANOVA tests or Kruskal-Wallis tests, for continuous variables. All analyses were two-tailed, and a $p$-value $\leq 0.05$ was considered statistically significant.

Predictors of LOS were evaluated using a stepwise linear regression model. LOS was treated as a continuous variable; however, the raw LOS variable was not normally distributed, we transformed the data using the natural logarithm (ln) which was normally distributed according to the Kolmogorov-Smirnov test $(p=0.200)$.

To identify potential confounding variables, we examined variables collected at baseline [i.e. gender, age at admission, ethnicity, relationship status, diagnosis, psychotic disorder diagnoses (schizophrenia, schizoaffective disorder, bipolar affective disorder, acute psychotic disorder)], a psychotic disorder with or without comorbid alcohol/substance misuse, referral source, previous PICU admission, interventions used during admission, number of seclusion episodes, number of restraint episodes, antipsychotic dose a \% of BNF maximum recommended daily dose, high-dose antipsychotic use defined as cumulative antipsychotic dose $>100 \%$ of BNF maximum recommended daily dose, antipsychotic polypharmacy use, use of mood stabiliser, benzodiazepines, depot antipsychotic medication, behaviour preceding referral to admission, assault, aggression, absconding, self-harm, multiple risks identified pre-admissions $(>1)$, the total number of risks identified, and forensic history. First, candidate variables were tested individually in a bivariate model; only variables with a coefficient of determination (R2) $>0.01$ (the variable explains, i.e. at least $1 \%$ of the variance in the outcome) and a $p$-value $<0.2$ on the bivariate model were included in the multivariable model.

The covariates with $\mathrm{R} 2>0.01$ and $p<0.20$ were considered for our multivariate model. We eliminated the variables with the largest $p$-values individually until all the remaining variables had $p<0.05$. This procedure highlighted gender, age at admission, acute psychotic disorder and schizoaffective disorder diagnoses, psychotic disorder with comorbid alcohol/substance misuse, number of restraint episodes, mood stabilisers, cumulative antipsychotic dose $>100 \%$ of maximum BNF recommended daily dose, multiple risks identified pre-admissions $(>1)$, as candidate variables for the final model. No collinearity was present with all VIF values between 1 and 2 .

\section{Results}

\section{Clinical characteristic of admissions}

Over the study period from January 2014 to January 2017 inclusive, there were 96 admission episodes to the PICU, with 91 admission episodes having complete demographic and clinical data (see Table 1). Five admission episodes had no clinical data and were excluded from the analysis. All patients were admitted under MHA legislation, and 31\% $(n=28)$ of admissions had a prior forensic history. Fifty-eight per cent $(n=53)$ of admission episodes were for men and $42 \%(n=38)$ were for women. The mean age was 37.1 (s.D. =11.3) (range 
Table 1. Sociodemographic and clinical characteristics of cases and gender comparisons (n; \%)

\begin{tabular}{|c|c|c|c|c|}
\hline & Total & Male $(n ; \%)$ & Female $(n ; \%)$ & $\chi^{2} / t$ test; $p$ value \\
\hline Gender & 91 & $53(58.2 \%)$ & $38(41.8 \%)$ & \\
\hline Age mean (S.D.) & $37.1(11.3)$ & $34.2(10.3)$ & $41.2(11.4)$ & 0.003 \\
\hline Ethnicity & & & & $\chi^{2}$ \\
\hline White & $82(90.2 \%)$ & $48(58.5 \%)$ & $34(41.5 \%)$ & $3.342 ; 0.342$ \\
\hline Black African & $4(4.4 \%)$ & $1(25.0 \%)$ & $3(75.0 \%)$ & \\
\hline Asian & $2(2.2 \%)$ & $2(100 \%)$ & 0 & \\
\hline Middle Eastern & $3(3.3 \%)$ & $2(66.7 \%)$ & $1(33.3 \%)$ & \\
\hline Marital status & & & & $\chi^{2}$ \\
\hline Single & $84(92.3 \%)$ & $50(59.5 \%)$ & $34(40.5 \%)$ & $2.853 ; 0.240$ \\
\hline Married/in a relationship & $5(5.5 \%)$ & $3(60.0 \%)$ & $2(40.0 \%)$ & \\
\hline Divorced/separated & $2(2.2 \%)$ & 0 & $2(100 \%)$ & \\
\hline Diagnosis $(n=; \%)$ & & & & $\chi^{2}$ \\
\hline Schizophrenia & $39(42.9 \%)$ & $31(79.5 \%)$ & $8(21.5 \%)$ & $22.823 ; p<0.001$ \\
\hline SAD & $18(18.0 \%)$ & $6(33.3 \%)$ & $12(66.7 \%)$ & \\
\hline BPAD & $21(21.0 \%)$ & $14(66.7 \%)$ & $7(33.3 \%)$ & \\
\hline Acute psychotic disorder & $9(9.0 \%)$ & $2(22.2 \%)$ & $7(77.8 \%)$ & \\
\hline Depression & 1 & 0 & $1(100 \%)$ & \\
\hline EUPD & $2(2.0 \%)$ & 0 & $2(100 \%)$ & \\
\hline ADHD & $1(1.2 \%)$ & 0 & $1(100 \%)$ & \\
\hline Psychotic disorder with the presence of alcohol/substances & $45(54.2 \%)$ & $33(73.3 \%)$ & $12(26.7 \%)$ & $\chi^{2}$ \\
\hline Psychotic disorder without alcohol/substances & $38(45.8 \%)$ & $20(52.6 \%)$ & $18(72.7 \%)$ & $3.826 ; 0.042$ \\
\hline
\end{tabular}

$\chi^{2}=$ chi-squared test; S.D., standard deviation.

18-63) (see Table 1). Majority of admissions $43 \%$ $(n=39)$ had a diagnosis of schizophrenia, followed by bipolar affective disorder BPAD $21 \%(n=21)$, schizoaffective disorder $18 \%(n=18)$ and acute psychotic disorder $9 \%(n=9)$.

\section{Risk factors leading to admission}

We identified assault as the primary risk factor for pre-admission $62 \%(n=62)$ to the PICU. Aggression pre-admission was $15 \%(n=14)$, and absconsion preadmission was $10 \%(n=9)$ in the number of admission to the PICU. Only $1.1 \%(n=1)$ of the risk factors leading to admission were to self-harm. Overall, $60 \%(n=55)$ of these patients had multiple risks identified for preadmission (see Table 2).

\section{Course of admission}

Seventy-four per cent $(n=39)$ of admissions did not require 1:1 nursing, seclusion or restraint use. Only $13 \%(n=7)$ of admissions had a seclusion episode and $6 \%(n=3)$ had restraint over the course of the 3 -year study period. The mean number of seclusion episodes was 2.12 (s.D. $=4.7$ ) (range $=0-28 ; n=90$ ) with an average number of restraints of 1.99 (S.D. $=4.7$; range $=0-27 ; n=89$ ).
Antipsychotic medication was used in $100 \%(n=91)$ of the admissions and antipsychotic polypharmacy drugs in $61 \%(n=55)$. High-dose antipsychotics were used in $68 \%$ $(n=15)$ of those admissions treated with single antipsychotic use. Sixty percent $(n=55)$ of the admissions were treated with antipsychotic polypharmacy, and $59 \%(n=54)$ were treated with a cumulative antipsychotic dose $>100 \%$ BNF maximum recommended daily dose. The mean daily antipsychotic dosage was $139.4 \%$ (S.D. $=65.1$ ) of BNF maximum daily dose. Males were more likely to be treated with antipsychotic polypharmacy $(n=39,74 \%)$ compared to females $(n=16,42 \%)$ $(x=9.173, p=0.002)$. A mood stabiliser was used in $44 \%(n=44)$ of the admissions and benzodiazepines for $70 \%(n=70)$.

\section{Length of stay (LOS)}

The mean LOS was 59.3 (S.D. =61.0) days, and the median was 39.5 days. Patients that were previously admitted measured a significantly longer LOS (mean $=83.3$ (s.D. $=87.2$ ) compared to those with a first PICU admission (mean $=48.9$ (S.D. $=42.3) \quad(t=2.524$, $p=0.013)$. Female gender $(B=0.826, p<0.001,95 \%$ CI: $0.450,1.201)$ and having more than one risk identified prior to admission $(B=0.401, p=0.011,95 \% \mathrm{CI}$ : $0.011,0.096)$ were associated with a longer LOS. 
Table 2. Pre-admission clinical characteristics

\begin{tabular}{|c|c|c|c|c|}
\hline & Total & Male $(n ; \%)$ & Female $(n ; \%)$ & $\chi^{2} / \mathrm{t}$ test; $p$ value \\
\hline Referral source & & & & $\chi^{2}$ \\
\hline Dublin area & $75(82.4 \%)$ & $45(84.9 \%)$ & $30(78.9 \%)$ & $0.542 ; 0.321$ \\
\hline Outside Dublin area & $16(17.6 \%)$ & $8(15.1 \%)$ & $8(21.1 \%)$ & \\
\hline Previous PICU admission & & & & $\chi^{2}$ \\
\hline Yes & $27(29.7 \%)$ & $13(24.5 \%)$ & $14(36.8 \%)$ & $1.608 ; 0.150$ \\
\hline No & $64(70.3 \%)$ & $40(75.5 \%)$ & $24(63.2 \%)$ & \\
\hline Reason for PICU admission & & & & $\chi^{2}$ \\
\hline Assault & $62(62.0 \%)$ & $37(59.7 \%)$ & $25(40.3 \%)$ & $1.017 ; 0.219$ \\
\hline Aggression & $14(15.4 \%)$ & $11(78.6 \%)$ & $3(21.4 \%)$ & $4.582 ; 0.032$ \\
\hline Absconding & $9(9.9 \%)$ & $4(44.4 \%)$ & $5(55.6 \%)$ & $0.733 ; 0.264$ \\
\hline Suicidal ideation/risk & $1(1.1 \%)$ & 0 & $1(100 \%)$ & \\
\hline No risk identified & $5(5.5 \%)$ & $1(20.0 \%)$ & $4(80.0 \%)$ & \\
\hline Multiple reasons identified for admission $(>1)$ & & & & $\chi^{2}$ \\
\hline Yes & $55(60.4 \%)$ & $30(56.6 \%)$ & $25(65.8 \%)$ & $0.781 ; 0.253$ \\
\hline No & $36(39.6 \%)$ & $23(43.4 \%)$ & $13(34.2 \%)$ & \\
\hline Forensic history & & & & $\chi^{2}$ \\
\hline Yes & $28(30.8 \%)$ & $20(37.7 \%)$ & $8(21.1 \%)$ & $2.892 ; 0.070$ \\
\hline No & $63(69.2 \%)$ & $33(62.3 \%)$ & $30(78.9 \%)$ & \\
\hline Number of risks identified pre-admission [mean (S.D.)] & $1.8(0.9)$ (range:0-4) & $1.8(1.0)$ & $1.7(0.9)$ & $\begin{array}{c}t \text { test } \\
0.22 ; 0.044\end{array}$ \\
\hline
\end{tabular}

$\chi^{2}$, chi-squared test; S.D., standard deviation.

Table 3. Associations between demographic and clinical variables and duration of admission

\begin{tabular}{|c|c|c|c|c|}
\hline & $\beta$ & $95 \% \mathrm{CI}$ & $95 \% \mathrm{CI}$ & p-value \\
\hline Gender & 0.826 & 2.606 & 3.919 & $<0.001$ \\
\hline Age & 0.005 & -0.110 & 0.019 & 0.500 \\
\hline Diagnosis of schizoaffective disorder & 0.142 & -0.273 & 0.556 & 0.497 \\
\hline Diagnosis of acute psychosis disorder & -1.207 & -1.691 & -0.363 & 0.003 \\
\hline Psychotic disorder with comorbid alcohol/substance misuse & -0.112 & -0.424 & 0.200 & 0.477 \\
\hline Multiple risks identified pre-admission $(>1)$ & 0.401 & 0.096 & 0.706 & 0.011 \\
\hline Number of restraint episodes & 0.021 & -0.019 & 0.061 & 0.295 \\
\hline Mood stabiliser used & -0.344 & -0.649 & -0.038 & 0.028 \\
\hline
\end{tabular}

$\beta$, beta coefficient; CIs, confidence intervals.

A diagnosis of an acute psychotic disorder $(B=-1.027$, $p=0.003,95 \%$ CI: $-1.691,-0.363)$ and the use of mood stabilizers $(B=-0.344, p=0.028,95 \% \quad C I:-0.649$, -0.038 ) were associated with reduced LOS in PICU (see Table 3). Those with a diagnosis of schizoaffective disorder had the longest average LOS (mean $=90.6$ $($ S.D. $=71.4)$ ), which was significantly longer than those with an acute psychotic disorder, who had a mean LOS of 20.4 (S.D. $=16.2)$ days $(F=2.985, p=0.036)$.

Each admission episode was associated with an average of 2.1 (S.D. $=4.7$ ) seclusion episodes and 2.0 (s.D. $=4.7)$ restraint episodes. Those requiring 1:1 nursing had a significantly increased LOS (mean 149.0 (s.D. $=171.3$ ) days) compared to patients who had other interventions or no interventions, including seclusion $($ mean $=52.1$ (S.D. $=55.0)$ days $)$ and physical restraint $($ mean $=43.3$ (S.D. $=22.5$ ) days; $F=4.426, p=0.08$ ).

\section{Discussion}

We report for the first time on clinical characteristics of patients admitted to a PICU in Ireland. In doing so, we contribute a number of significant findings to the limited extant research in clinical care in PICUs.

Our study provides one of the most detailed reports of medication use and other management interventions during PICU admissions. Few PICU studies have reported on medication use (Goldney et al. 1985; Musisi SM et al. 1989; 
Brown \& Bass, 2004; Hochstrasser et al. 2018; Winkler et al. 2019), and few have provided detailed analysis. Our study demonstrated that antipsychotic polypharmacy was commonly used in treatment (see Table 2). An interesting development describing males were less likely to be treated with single high-dose antipsychotics $(n=11,65 \%)$ compared to females $(n=4,80 \%)$. Differing multidisciplinary team practices could be a contributing factor. This study described high-dose antipsychotic use measured by cumulative antipsychotic dose $>100 \%$ of BNF maximum recommended daily use. This finding highlights the severity and complexity of this cohort of patients which was prudent in the administration of high-dose antipsychotic medication.

Assaultive behaviour is a typical risk behaviour preceding PICU admission. However, over the course of admission, the low incidence of violence at the PCC reflects similar studies (Crowhurst \& Bowers, 2002; Bowers \& Cullen, 2017). The PCC had reduced the risk and need for seclusion (13.2 \%) and restraint (5.7\%) compared to secondary acute psychiatric units. This could be due to the built for purpose facility including the higher nursing staff ratio and low stimulus environment (Wynaden \& Mcgowan, 2001; Vaaler et al., 2006; Bailey, 2014; Health Service Executive, 2015). Meaningful activity programmes including gardening, table tennis, gym exercise, artwork and baking facilitated by nursing staff and the occupational therapist substantially reduced the level of agitation and physical aggression of patients on the ward.

An important finding from our study was the shorter average LOS for males compared to females. A recent UK retrospective cohort identified a similar shorter average LOS for male patients, with an average overall LOS of 43 days (O'Brien et al. 2013). To the best of our knowledge, our study is the first to replicate this finding. Our average LOS of 59 days was longer than that reported in most other studies (Bowers et al. 2008). The unique nature of our PICU, in that it is a stand-alone hospital site, and the lack of integration with a general hospital or general adult psychiatry hospital setting may have contributed to the longer average duration of stay. In contrast to most other studies, we provide a median LOS of 40 days. This is still longer than average PICU LOS of 1-2 weeks (Bowers et al. 2008). The use of a median LOS provides a more accurate LOS measure, by excluding outlier longer stay patients. The different MDT management in the two units is accountable for the difference in LOS between male and female patients. Patients that had previous admissions to the PICU had longer LOS reflecting previous studies (Thompson EE et al. 2003). LOS was extended in patients requiring a 1:1 special compared to those who had a history of absconsion, violence, incidence of seclusion or physical restraint (Vaaler et al. 2006).
Similar to other studies, the majority of PICU admissions had a diagnosis of schizophrenia or schizoaffective disorder (Bowers et al. 2008; O'Brien et al. 2013). We identified that schizophrenia was the largest diagnostic category, accounting for $46 \%$ of patients. A review of PICU admissions found that in UK settings that schizophrenia accounts for $50 \%$ of admissions, with a further $20 \%$ admitted with mania (Bowers et al. 2008). We identified the low rate of personality disorders as a primary diagnosis, similar to previous studies (Winkler et al. 2019).

We identified a PICU readmission rate of $30 \%$ over three-year follow-up period. Prior studies with shorter follow-up periods of approximately one year have identified rates of PICU readmission ranging from 9\% (Lee et al. 2000) to 30-35\% (Warneke, 1986; Mitchell, 1992; Eaton \& Ghannon-2000; Brown \& Bass, 2004) and as high as $66 \%$ (Pereira et al,. 2006) within one year follow-up. Patients in these units benefit from profile-specific interventions before, during and after any incident that might occur (Hochstrasser et al. 2018). This would be achievable due to the higher nursing to patient ratio (1 nurse: 2 patient) that leads to more one to one staff time with patients in PICUs compared to an acute psychiatric unit (Clinton \& Pereira, 2001; Gwinner \& Ward, 2013).

Acute psychotic disorder, male gender and the use of mood stabilisers were all associated with shorter PICU LOS (Table 3). In the broader psychosis literature, overall LOS in the hospital has reduced in the years of follow-up after first episode psychosis (FEP), with people spending an average of 107 days hospitalised over a seven-year follow-up period (with over half of all FEP cases being hospitalised in the first seven years of illness) (Ajnakina et al. 2019). Recovery rates remain suboptimal in schizophrenia (Lally et al. 2017), with high rates of treatment-resistant schizophrenia existing from early stages of psychosis (Lally et al. 2016). PICU admissions can be required components of hospital admissions for some people with schizophrenia, indicative of a more severe symptom profile, but also heightened risk of aggression and violence.

\section{Strengths and limitations}

A strength in this study was the comprehensive data collection, clinical information and a large data set relevant to previous studies in these settings. We have provided a quasi-prospective cohort analysis of the clinical course of PICU admission for a relatively large sample, in comparison to other work in this area (GintalaiteBieliauskiene et al. 2011; Winkler et al. 2011).

This study was conducted in a single-site PICU, in a hospital setting remote from general hospital or general adult psychiatric hospital settings. This may limit the 
generalisability of our findings. We have no data on how aggression or violence was defined, and this may have varied across referring to hospital sites. However, we were able to examine a wide range of pre-admission risks, and thus provide a reasonably comprehensive review of pre-admission behaviours. We do not have information on broader service level factors that may have affected decisions to transfer patients to PICU. Some of the primary hospital sites may not have had access to seclusion facilities, thus altering the threshold for admission across different hospital sites.

A limitation is the lack of use of a structured objective scale such as the Clinical Global Impression (CGI) rating scale by MDT members. The use of a CGI rating scale may have provided us with the opportunity to measure symptom severity and treatment response and efficacy in our patient cohort. However, we provided data on comorbid alcohol or substance use disorders, with no evidence of increased LOS with comorbid substance use in those with psychotic disorders.

Another limitation is the lack of data on patient perspectives on their PICU admission. There is now an increased drive in psychiatry to lessen unnecessary coercive treatment (Mielau et al. 2018), and assessing patient satisfaction and opinion on care received in the PICU setting would be an important consideration in improving this.

\section{Conclusion}

PICU is an essential service for the severely ill psychiatric patients and is a progressively developing sub-speciality. Our research findings provide novel insights into clinical care in this PICU setting. We determined that physical assaults are an important determinant in PICU admission. The PICU setting provides clinical management and intensive care to the most disturbed psychiatric patients. In this study, we provide important evidence for the characteristics of treatment and duration of admission. Further research is needed to expand on these findings in moving forward with the delivery of high standard care in PICUs.

\section{Acknowledgements}

The authors wish to acknowledge the assistance of Ray Farrelly and Anne Marie Lynch in particular for their help.

\section{Financial Support}

This research received no specific grant from any funding agency, commercial or not profit sectors.

\section{Conflicts of interest}

None.

\section{Ethical standards}

This author asserts that all procedures contributing to this work comply with the ethical standards of the relevant national and institutional committee on human experimentation with the Helsinki declaration of 1975, as revised in 2008. The authors assert that their local Ethics Committee granted ethical approval and GDPR for publication of this retrospective study.

\section{References}

Ajnakina O, Stubbs B, Francis E, Gaughran F, David AS, Murray RM, Lally J (2019). Hospitalisation and length of hospital stay following first-episode psychosis: systematic review and meta-analysis of longitudinal studies. Psychological Medicine 6, 1-11.

Bailey DS (2014) National Minimum Standards for Psychiatric Intensive Care in General Adult Services NAPICU.

Barlow K, Grenyer B, Ilkiw-Lavalle O (2000). Prevalence and precipitants of aggression in psychiatric inpatient units. Australian and New Zealand Journal of Psychiatry 34, 967-974.

Basson JV and Woodside M (1981). Assessment of a secure/intensive care/forensic ward. Acta Psychiatrica Scandinavica 64, 132-141.

Bowers L and Cullen A (2017). Seclusion and Psychiatric Intensive Care Evaluation Study (SPICES): combined qualitative and quantitative approaches to the uses and outcomes of coercive practices in mental health services. Health Services and Delivery Research 5, 1-116.

Bowers L, Jeffery D, Bilgin H, Jarrett M, Simpson A, Jones J (2008). Psychiatric Intensive Care Units: a literature review. The International Journal of Social Psychiatry 54, 56-68.

Brown S, Bass N (2004). The psychiatric intensive care unit: patient characteristics, treatment and outcome. Journal of mental Health 13, 601-609.

Clinton C, Pereira S (2001). Training needs of psychiatric intensive care staff. Nursing Standard 15, 33-36.

Crowhurst N, Bowers L (2002). Philosophy, care and treatment on the psychiatric intensive care unit: themes, trends and future practice. Journal of Psychiatric and Mental Health Nursing 9, 689-695.

Eaton S, Ghannon M (2000). Prediction of violence on a PICU. Medicine, Science and the Law 40, 143-146.

Gintalaite-Bieliauskiene K, Tandon V, Woochit V (2011). Female-only psychiatric intensive care unit (PICU): Prospective study of patient demographics and outcomes at Elizabeth Casson House, Bristol, UK. European Psychiatry 26, 1875.

Goldney R, Bowes J, Spence N, Czechowicz A, Hurley R (1985) The psychiatric intensive care unit. British Journal of Psychiatry, 146(1), 50-54. doi: 10.1192/bjp.146.1.50

Gwinner K and Ward L (2013). P.I.C.U., H.D.U., A.O.A What treatment do we provide? Current descriptions 
of the function of intensive care for inpatient psychiatric health care. Mental Health Review Journal 18, 128-143.

Hochstrasser L, Voulgaris A, Möller J (2018). Reduced frequency of cases with seclusion is associated with "opening the doors" of a Psychiatric Intensive Care Unit. Frontiers in Psychiatry 9, 57.

HSE.ie. (2015). Phoenix Care Centre - HSE.ie. (https:/ / www.hse.ie/eng/services/list/1/lho/dublinnw/mentalhealth-services/phoenix-care-centre/)

HSE.ie. (2017). A Vision for Change - Mental Health and Suicide Prevention - HSE.ie. (https:/ /www.hse.ie/eng/ services/publications/mentalhealth/visionforchange.html)

Kessler RC, Demler O, Frank RG, Olfson M, Pincus HA, Walters EE,.Wang P, Wells KB and Zaslavsky AM (2005). US prevalence and treatment of mental disorders: 19902003. New England Journal of Medicine 352, 2515-2523.

Koppelmans V and Schoevers R (2009). The Amsterdam Studies of Acute Psychiatry - II (ASAP-II): a comparative study of psychiatric intensive care units in the Netherlands. BMC Public Health 9.

Lally J, Ajnakina O, Di Forti M, Trotta A, Demjaha A, Kolliakou A, et al. (2016) Two distinct patterns of treatment resistance: clinical predictors of treatment resistance in first-episode schizophrenia spectrum psychoses. Psychological Medicine 46, 3231-3240.

Lally J, Ajnakina O, Stubbs B, Cullinane M, Murphy KC, Gaughran F, Murray RM (2017) Remission and recovery from first-episode psychosis in adults: systematic review and meta-analysis of long-term outcome studies. British Journal of Psychiatry 211, 350-358.

Lee J, Schwartz D, Hallmayer J (2000). Catatonia in a psychiatric intensive care facility: incidence and response to benzodiazepines. Annals of Clinical Psychiatry 12, 89-96.

Mielau J, Altunbay J, Lehmann A, Bermpohl F, Heinz A, Montag C (2018). The influence of coercive measures on patients' stances towards psychiatric institutions.

International Journal of Psychiatry in Clinical Practice 22, 115.

Mitchell GD (1992). A survey of psychiatric intensive care units in Scotland. Health Bulletin 50, 228-232.

Mounsey N (1979). Psychiatric intensive care. Nursing Times 75, 1811-1813.

Musisi SM, Wasylenki DA, Rapp MS (1989). A Psychiatric Intensive Care Unit in a Psychiatric hospital. The Canadian Journal of Psychiatry 34, 200-204.

O'Brien A, Cramer B, Rutherford M, Attard D (2013). A retrospective cohort study describing admissions to a London Trust's PICU beds over one year: do men and women use PICU differently? Journal of Psychiatric Intensive Care 9(1), 33-39.

Pararajasingam SR, Browne R, Bawagan A, Pauline T, Firdouas M, Nwarie E, Navanathan S (2017) Referral patterns to Psychiatry Intensive Care Unit: phoenix care centre. International Journal of Integrated Care 17, A600.

Pereira S, Sarsam S, Bhui K, Paton C (2006). The London Survey of Psychiatric Intensive Care Units: psychiatric intensive care; patient characteristics and pathways for admission and discharge. Journal of Psychiatric Intensive Care 1, 17-24.

Rachlin S (1973) On the need for a closed ward in an open hospital; the psychiatric intensive care unit. Hospital $\mathcal{E}$ Community Psychiatry 24, 829-833.

Robert G, Bowes J, Spence N, Czechowicz A, Hurley R (1985). The Psychiatric Intensive Care Unit. British Journal of Psychiatry 146, 50-54.

Thompson EE, Neighbors HW, Munday C, Trierweiler S (2003). Length of stay, referral to aftercare, and rehospitalization among psychiatric inpatients. Psychiatric Services 54, 1271-1276.

Vaaler A, Iversen V (2011). Short-term prediction of threatening and violent behaviour in an Acute Psychiatric Intensive Care Unit based on patient and environment characteristics. BMC Psychiatry 11.

Vaaler AE, Morken G, Fløvig J Chr., Iversen VC, Linaker OM (2006) Effects of a psychiatric intensive care unit in an acute psychiatric department, Nordic Journal of Psychiatry, 60:2, 144-149, doi: 10.1080/080394 80600583472

Vaaler AE, Morken G, Fløvig JC, Iversen VC, Linaker OM (2006). Effects of a psychiatric intensive care unit in an acute psychiatric department, Nordic Journal of Psychiatry 60, 144-149.

Warneke L (1986). A psychiatric intensive care unit in a general hospital setting. Canadian Journal of Psychiatry 31: 834-837.

Winkler D, Kaltenboeck A, Frey R, Kasper S, Pjrek E (2019). Changes over time of the diagnostic and therapeutic characteristics of patients of a psychiatric intensive care unit in Austria. Comprehensive Psychiatry 93, 20-26.

Winkler D, Naderi-Heiden A, Strnad A, Winkler-Pjrek E, Scharfetter J, Kasper S, Frey R (2011). P03-593 - The viennese psychiatric intensive care unit - diagnostic distribution. European Psychiatry, 0924-9338.

Wynaden D and Mcgowan S (2001). Types of patients in a Psychiatric Intensive Care Unit. Australian \& New Zealand Journal of Psychiatry 35, 841-845. 